\title{
Design and Performance Analysis of Anti-Surge Control Mechanism for Compressor System using Neural Networks
}

\author{
Divya M.N ${ }^{1}$ \\ Research Scholar \\ VTU Research Centre, MSRIT \\ VTU, Belagavi, India \\ Narayanappa C.K ${ }^{2}$ \\ Dept. of Medical Electronics
}

M.S. Ramaiah Institute of Technology, Bangalore, India

\author{
S L Gangadhariah ${ }^{3}$ \\ Dept. of Electronics and Communication \\ M.S. Ramaiah Institute of Technology \\ Bangalore, India
}

\author{
V Nuthan Prasad ${ }^{4}$ \\ Dept. of Electronics and Communication Organization \\ M.S. Ramaiah Institute of Technology, Bangalore, India
}

\begin{abstract}
The compressor system is caused by the surge, which is an instability occurrence in most gas-process and oil industries. These issues are solved by using a recycle valve that avoids the surge and provides higher mass flow in the compressor system. An advanced controller-based anti-surge control mechanism is a need in the compressor system to improve the stability and surge issues. In this manuscript, an efficient, Neural-network predictive controller (NNPC) based variable speed compressor recycle system is modeled with an anti-surge control mechanism. When the mass flow is deficient, the recycle system is introduced, acts as a safety system, and feeds the compressed gas back to the upstream system. The different controllers like Proportional Integral Derivative (PID) controller, Fuzzy logic controller (FLC), and Neuro-fuzzy controller (NFC) based anti-surge control mechanism are also used in Compressor recycle system to compare the stability and performance metrics with NNPC. The NNPC based compressor system provides a better operating position and dynamic response with less error than other controllers-based compressor systems.
\end{abstract}

Keywords-Anti-surge; fuzzy logic controller (FLC); neurofuzzy controller (NFC); compressors; neural-network

\section{INTRODUCTION}

The centrifugal Compressor is used in most industries like oil, aero-space, and gas-plant to increase pressure and oil production. The gas compressors are generally divided into four types: Axial-flow, centrifugal, rotary, and Reciprocatingtype. The rotary and Reciprocating-type compressors reduce the gas volume's occupancy and later apply the higher Pressure to discharge the gas. The axial and centrifugal compressors act as turbo-compressor with continuous flow [1-2]. The Axial compressors with rotating stall and surge are presented with theoretical and mathematical concepts by Greitzer et al. [3]. The non-dimensional parameter (B) factors define whether the Compressor is in surge or rotating stall conditions. If the ' $\mathrm{B}$ ' factor is lower, then a rotating stall will occur, which causes the instabilities by reducing the mass flow by rising the Pressure.
Similarly, a surge will occur with the mass flow and pressure oscillations if the' $\mathrm{B}^{\prime}$ factor is higher. The spool dynamics are introduced in the ' $\mathrm{B}$ ' parameter to improve the speed control [4]. The Full-range Surge and rotating-stall avoidance by Badmus et al. are presented in the compressor system. The model uses open-loop feed-forward and closedloop feedback control mechanisms in the throttle valve area to control the Pressure and mass flow. These control mechanisms are scheduled and use in rotating stall and surge avoidance [5]. The calculation of operating location between surge and the stable condition is necessary to avoid the surge in Compressor. To counteract the surge, the invariant coordinate system is introduced by changing molecular weight [6]. Gravdahl et al. present the drive-torque actuation system to regulate the active surge in the centrifugal Compressor. The active surge stabilization utilizing control law [7] causes the throttle line to appear left to the surge line.

In contrast, the compressor system for active surge control is presented by bohagen et al. using drive torque as a control input. The desired operating point stabilization is achieved with static and dynamic feedback mechanisms [8]. The Fuzzy logicbased surge control mechanism [9] is introduced for the Moore-Greitzer model with constant speed in the compressor system, stabilizing the different operating conditions and extending the stable line next to the surge line. The active surge control with variable speed mechanism [10] in centrifugal Compressor is designed to analyze the different performance metrics with better improvement than the Gravdahl et al. [1] work. The Contribution of the research work is organized as follows:

- The complete variable speed-based Compressor system with a recycling loop is modeled to ensure higher mass flow and avoid the surge.

- The Neural-network predictive controller (NNPC) based anti-surge control mechanism is modeled for the Compressor recycle system to improve stability and prevent active surges. 
- The different controllers like PID controller, Fuzzy logic controller (FLC), and Neuro-fuzzy controller (NFC) are also modeled in anti-surge control mechanism to analyze the stability and performance comparison.

In this manuscript, an efficient Compressor recycle system with an anti-surge control mechanism using NNPC is modeled and compared with other controllers to evaluate performance metrics. The manuscript is organized as follows: Section II explains the Compressor-based system's current works for different applications. The simple compressor system with mathematical expressions is described in Section III. The Compressor recycle system with an anti-surge control mechanism using other controllers is explained in Section IV. The results and discussion is carried out in Section V. Section VI concludes the overall work with performance improvement and also suggest the future scope.

\section{RELATED WORK}

This section analyzes the recent works on Compressor based systems for different application requirements. Budinis et al. [11-12] describe the Centrifugal compressor's control mechanism using a Model predictive controller (MPC), which can control the Compressor's Pressure during surge operation. The hot and cold recycle line is configured with an anti-surge control mechanism to analyze the performance metrics and power consumption. Cortinovis et al. [13-14] present the Linearize MPC-based centrifugal gas compressor system with an anti-surge and process control mechanism. These mechanisms are adopted in safe and electric-driven applications with improved surge $11 \%$ by maintaining distance and $50 \%$ reduction in process control time (settling). Sheng et al. [15] describe the Throttle and closed-coupled Valve (CCV) actuators based Compressor system for the instability control mechanism. The double actuators scheme is introduced to improve the control performance in CCV by tuning the Bparameter in the Throttle valve. Gritli et al. [16] present the Electronic throttle valve (ETV) optimization using a genetic algorithm (GA) by tuning the PID-based Fuzzy factors. The GA with PID-based FLC provides better disturbance rejection and trajectory tracking points than other conventional ETV approaches. Azeem et al. [17] present the ETV with FLC based super-twisting-Sliding mode control (STSMC) scheme. The STSMC scheme improves the controller's dynamic performance in ETV without compromising the tracking accuracy and stability features. Saeed et al. [18] present the Hybrid electric vehicles (HEVs) speed control mechanism using different controllers. The state observer controller (OBC) and linear quadratic regulator (LQR) based scheme provide better dynamic control response than the conventional PID Controller scheme in HEV.

The Neuro-Fuzzy controller (NFC) based Grid-connected gas turbine is modeled by Iqbal et al. [19] to improve the transient response and maintain stable operations. Liying et al. [20] present the non-linear compensation control mechanism using a PID controller for ETV to analyze the response time and performance. Zaibari et al. [21] explain the constant-speedbased centrifugal Compressor using Tube-MPC to control the surge instability. The Adaptive fuzzy logic with MPC acts as an anti-surge controller to avoid the surge and improve the robustness against the mass flow and pressure-based disturbances. Khsheem et al. [22] present the Surge compressor system with an active control mechanism. The PID controller controls the surge line and provides the dynamic response to use in the control valve. Guan et al. [23] present the centrifugal compressor surge control mechanism. The work analyzes the variable -tip clearance mechanism is introduced to control the surge and analyzes the compressor performance. The Surge control with different speeds and also with different throttle opening sizes are analyzed with simulation results. Aribi et al. [24] present the Recycle compressor system for active surge control using a hybrid adaptive controller. The PID with auto tuning Fuzzy interface system (FIS) controls the operating points under the Surge line at different speeds. The artificial neural network (ANN) based Centrifugal compressor is modeled by Ebrahimi et al. [25] to predict operational parameters.

\section{COMPRESSOR MODEL}

The surge control and instabilities are the main issues in any of the axial and centrifugal compressors. In this work, variable-speed Centrifugal-based Compressor is discussed. The overview of the Compressor system is represented in Fig. 1. It mainly contains a Torque drive, Compressor with duct, Plenum, and Throttle valve. The Compressor model produces the mass and Pressure using torque drive and plenum units. The Throttle valve delivers the mass throttle value and feedback to the Plenum unit and Compressor mass flow.

When the mass is applied to the Plenum unit, which produces the Pressure, it is represented in Equation (1). The momentum applied on the Compressor with duct produces the mass flow is described in Equation (2). The torque drive delivers the angular speed using the angular momentum feature represented in Equation (3). The work was initially presented by Gravdahl et al. [1], and it is extended by Fink et al. [2]. The Pressure, mass flow, and speed is represented in Equation (1-3) as follows:

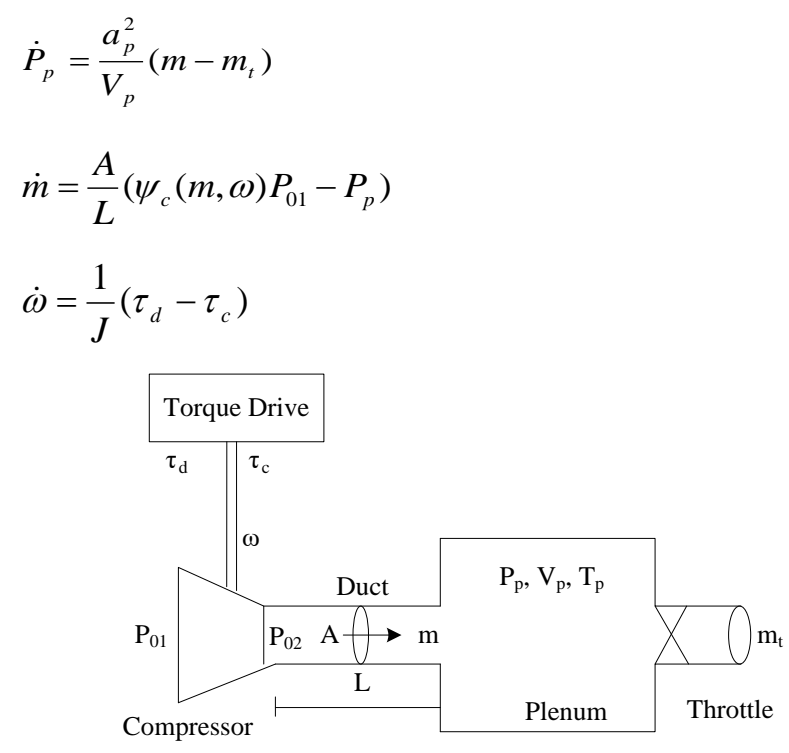

Fig. 1. An Overview of the Compressor System. 
The above Equations (1-3) uses few of the notations as follows: where $\dot{m}$ is mass flow, $\dot{P}_{p}$ is Plenum Pressure, $\omega$ is Torque speed of the impeller, $V_{p}$ is Plenum Volume, $a_{p}$ Plenum's speed of the gas, $\mathrm{m}_{\mathrm{t}}$ is Throttle's mass flow, $A$ is the cross-sectional area of the duct, $L$ is the length of the duct, $P_{01}$ is the Pressure (Ambient), $\psi_{c}$ is Compressor Characteristic, $J$ represents the moment of inertia of torque, $\tau_{d}$ is Torque drive, and $\tau_{c}$ is impeller blades torque.

The predicting surge is possible using Equations (1-3) and is one-dimensional in centrifugal compressors. When Compressor is in the deep surge point, and the impeller blade's torque is represented in Equation (4) as follows:

$\tau_{c}=|m| r_{2}^{2} \mu \omega$

The Compressor system is used to determine the pressure ratio using $\psi_{\mathrm{c}}$. The compressor characteristic is expressed in Equation (5) as follows [1]:

$\psi_{c}(m, \omega)=\left(1+\frac{\mu r_{2}^{2} \omega^{2}-\frac{1}{2} r_{1}^{2}(\omega-\alpha m)^{2}-k_{f} m^{2}}{a_{p} T_{1}}\right)^{\frac{k}{k-1}}$

The throttle mass flow is represented in Equation (6) as follows:

$m_{t}=\tanh \left(\varsigma\left(P_{p}-P_{01}\right)\right) A_{t} \sqrt{\left(P_{p}-P_{01}\right) \tanh \left(\varsigma\left(P_{p}-P_{01}\right)\right)}$

The above Equations (4-6) uses a few of the notations as follows: $r_{1}$ is inducer radius, and $r_{2}$ is impeller exit radius, $\mu$ is flow co-efficient, $k$ is heat capacity ratio, $T_{1}$ is inlet temperature, $A_{t}$ is Orifice Opening area, and $\zeta$ is zeta $(>>1)$. The Compressor characteristic provides the performance metrics of the Compressor using mass flow and Pressure. When the mass flow decreases, the Pressure will increase until its instability point, Then Surge or rotating stall problems arise in the compressor system.

\section{COMPRESSOR With ANTI-Surge CONTROL USING DIFFERENT CONTROLLERS}

The oscillations, stalls/surge, and lower pressure rises will always affect the centrifugal Compressor's performance. The operating point is always far away from the surge line (SL), which is the best option at low pressure and high mass flow conditions. But the efficiency of the Compressor always lacks in this region. So for better efficiency, the Pressure will rise at its highest, which is close to the SL. Surge avoidance is also known as an anti-surge control mechanism. Most commonly, the recycle valves are used to avoid the surge in industry applications. The Compressor Recycle system based Antisurge Control Mechanism using different Controllers is represented in Fig. 2.

Using an anti-surge control mechanism, the Compressor recycle system contains a Suction unit, Compressor with duct, Torque drive unit, Plenum and recycle valve units. The feed flow mechanism is essential to analyze the compressor system performance if the feed flow value is high or too low, which affects the operating point and speed features in the compressor system. Suppose the pressure $P_{1}$ value is too high in the suction unit, which fails to operate in the compressor system. The Suction unit with Pressure $\left(\mathrm{P}_{1}\right)$, and Volume1 $\left(\mathrm{V}_{1}\right)$, are part of the upstream piping operation. Similarly, The Plenum unit with Pressure $\left(\mathrm{P}_{2}\right)$, and Volume2 $\left(\mathrm{V}_{2}\right)$, are part of the downstream piping operation. The modeling of the Compressor recycle system is represented in Fig. 3.

The amount of feed flow $\left(\mathrm{m}_{\mathrm{f}}\right)$ with mass applied on the Suction unit, which generates the Pressure $\left(\mathrm{P}_{1}\right)$ and is represented in Equation (7). The mass flow is used on the Plenum unit, which produces the Pressure $\left(\mathrm{P}_{2}\right)$ and is represented in Equation (8). The momentum applied to the Compressor creates the mass flow (m), described in Equation (9). The throttle mass flow of the compressor recycle system is represented in Equation (10) as follows:

$$
\begin{aligned}
& \dot{P}_{1}=\frac{a_{p}^{2}}{V_{1}}\left(m_{f}-m_{r}-m\right) \\
& \dot{P}_{2}=\frac{a_{p}^{2}}{V_{2}}\left(m-m_{t}-m_{r}\right) \\
& \dot{m}=\frac{A}{L}\left(\psi_{c}(m, \omega) P_{1}-P_{2}\right) \\
& m_{t}=\left.\tanh \left(\varsigma\left(P_{2}-P_{01}\right)\right) C_{t} \sqrt{\left(P_{2}-P_{01}\right) \tanh \left(\varsigma\left(P_{2}-P_{01}\right)\right)}\right|_{\varsigma>0}
\end{aligned}
$$

The feed flow mechanism is dependent more on the type of Compressor used and is not constant while performing the simulation process. The feed flow $\left(\mathrm{m}_{\mathrm{f}}\right)$ operation is represented in Equation (11), and recycle flow $\left(\mathrm{m}_{\mathrm{r}}\right.$ ) operation is described in Equation (12) and are as follows:

$$
\begin{aligned}
& m_{f}=C_{f} \sqrt{P_{01 u}-P_{1}} \\
& m_{r}=C r \sqrt{P_{2}-P_{1}}
\end{aligned}
$$

$\mathrm{C}_{\mathrm{f}}$ and $\mathrm{C}_{\mathrm{r}}$ are coefficients of Feed flow and recycle flow, respectively; $\mathrm{P}_{01 \mathrm{u}}$ is upstream ambient Pressure.

The Anti-Surge Control mechanism using PID Controller with different Controllers like Fuzzy logic controller (FLC), Neuro-fuzzy Controller (NFC), and Neutral network Predictive Controller (NNPC) are represented in Fig. 4. In this work, the PID controller is used as the industry standard and other controllers to analyze the anti-surge control operations. The Compressor system produces the mass flow (m) and compressor characteristic $\left(\psi_{\mathrm{c}}\right)$ outputs and inputs the anti-surge mechanism. The different controller's output $\left(a s c_{i n}\right)$ is input the recycle value unit. The Surge control line (SCL) is considered along with the surge line (SL), which is linear and horizontal to the surge margin. The compressor characteristic SCL with the mass flow is represented in Equation (13) as follows:

$$
\psi_{s c l}(m)=i m+j
$$




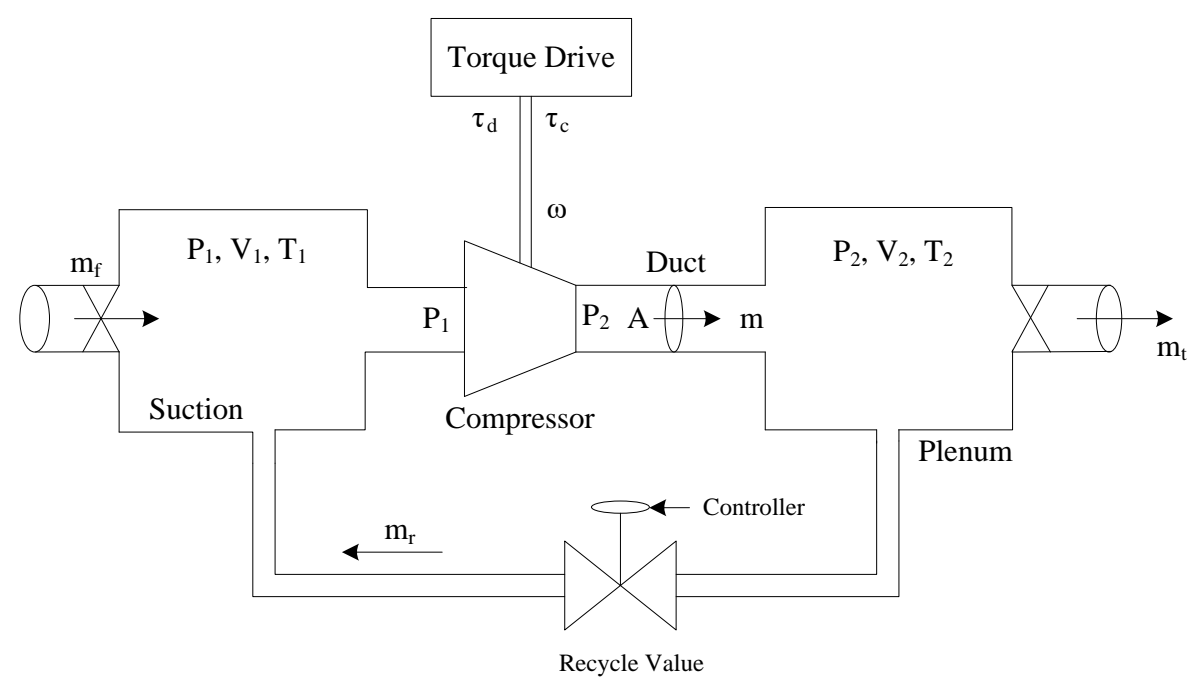

Fig. 2. Compressor Recycle System based Anti-surge Control Mechanism in using different Controllers.

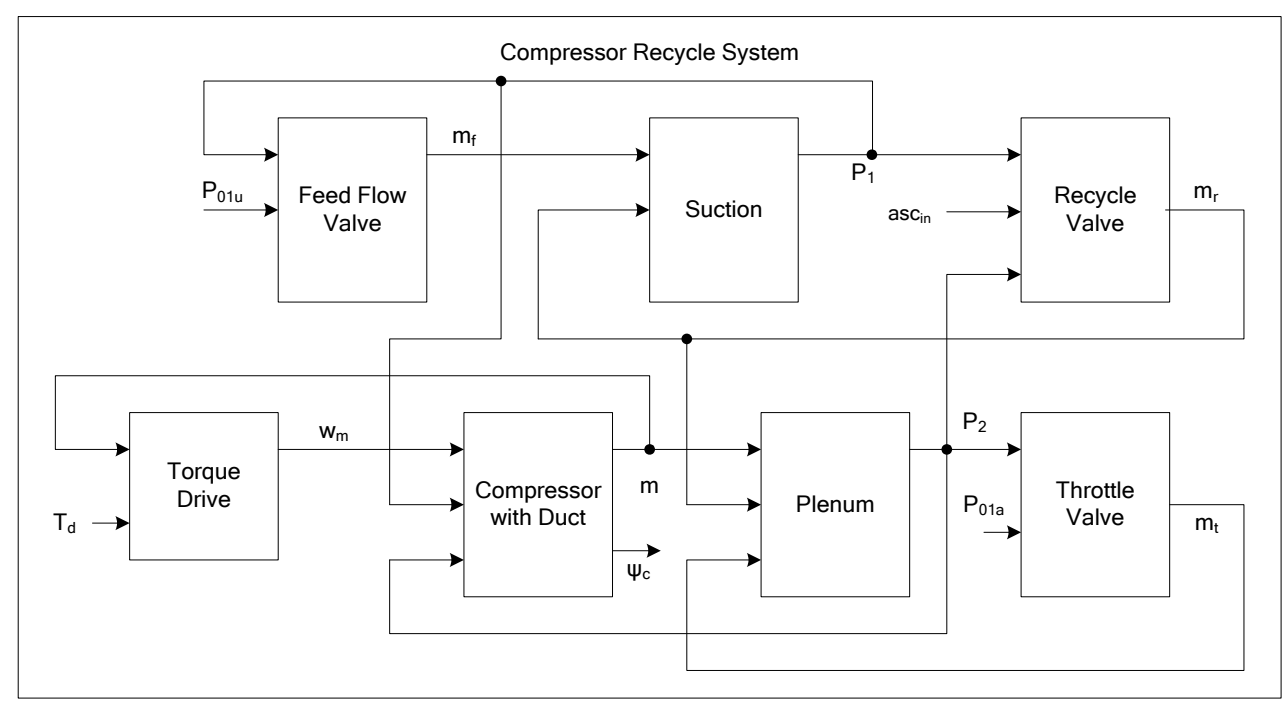

Fig. 3. Modeling of the Compressor Recycle System.

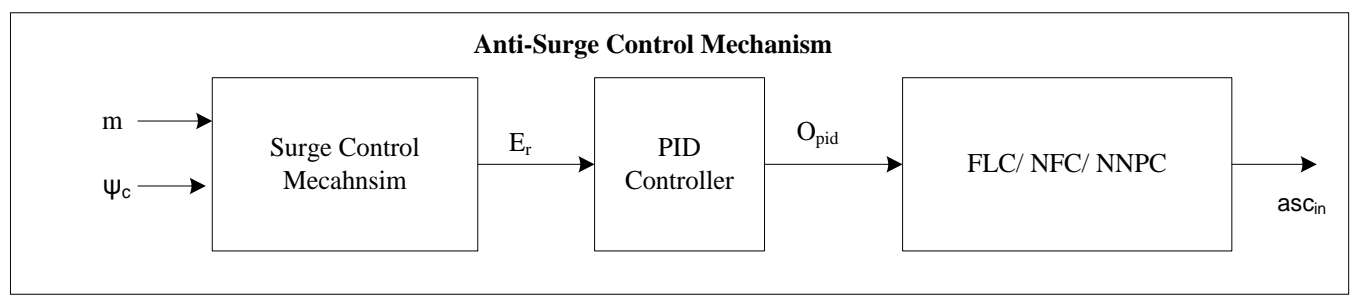

Fig. 4. Anti-Surge Control Mechanism using different Controllers.

Where $\mathrm{i}$ and $\mathrm{j}$ are the coefficients for SCL, when the operating point (OP) is right to the SCL, the control mechanism is not activated.

Only when the operating point (OP) is left to the SCL, the control mechanism is activated. The PID controller and other controllers (FLC/ NFC/NNPC) are used to getting the OP back to the right, with its controlling mechanism. To analyze the controlling mechanism, first, find the distance $\left(\mathrm{d}_{\mathrm{i}}\right)$, as the difference (horizontal) between the SCL and OP, which is represented in Equation (14) as follows:

$$
d_{i}=m-m_{s c l}\left(\psi_{c}\right)
$$

The OP is calculated using mass (m) and $\psi_{\mathrm{c}}$. The $\Psi_{\mathrm{scl}}(\mathrm{m})$ is same as $\psi_{\mathrm{c}}$, when Pressure rises and consider the inverse of Equation (13) to calculate the mscl $\left(\psi_{\mathrm{c}}\right)$, which is represented in Equation (15) as follows:

$m_{s c l}\left(\psi_{c}\right)=\frac{\psi_{c}-j}{i}$ 
Use Equation (15) to calculate the distance value in Equation (14). The error value is calculated based on the horizontal distance using OP and SCL. When the distance value is +ve, the error value $\left(E_{r}\right)$ is set to zero, and no need to perform any controlling operation, and the $\mathrm{OP}$ is located to the right position of SCL. When the distance value is -ve, its +ve value is used to calculate the error value $\left(E_{r}\right)$ using controlling operation. The error value $\left(E_{r}\right)$ calculation is represented in Equation (16) as follows:

$E_{r}=\left\{\begin{array}{cc}0 & d_{i}>0 \\ -d_{i} & \text { else }\end{array}\right.$

This error value $\left(E_{r}\right)$ is input to the PID controller and it is represented in Equation (17) as follows:

$O_{p i d}=K_{p} E_{r}+K_{i} \int_{0}^{t} E_{r} d t$

Where $K_{p}$ and $K_{i}$ are the proportional gain and integral gain of the PID controller, the PID Controller output $\left(\mathrm{O}_{\text {pid }}\right)$ is used directly to control the flow percentage in recycling valve for the anti-surge control mechanism. To improve the antisurge control with OP, a different controlling mechanism is introduced.

\section{A. Fuzzy Logic Controller (FLC)}

The Fuzzy logic Controller (FLC) provides a better dynamic response than the conventional PID controllers and is used in most industrial control applications. The FLC mainly contains five blocks: the Compressor recycle system: Fuzzification, De-fuzzification, database (Knowledge base), Ruleset, and evaluation process. The Fuzzification converts absolute (crisp) data into Fuzzy (linguistic) data. The PID controller output is input to the fuzzification process as crisp data and produces the fuzzy data. These fuzzy data values are considered as Fuzzy-sets in FLC. The membership functions analyze these fuzzy sets and estimate whether they are low, high, or oversized. The evaluation process provides the decision to control and study the fuzzy rules stored in the rule base. The FLC process performance is analyzed by rule base. The de-fuzzification process converts back the Fuzzy data into crisp data. The Knowledge-based is used to store the membership functions of both the Fuzzification and defuzzification processes. The evaluation of fuzzy rules is done by basic fuzzy sets with AND, OR, and NOT operations. The de-fuzzification output is the final FLC output and is processed in Compressor recycle system. The function of the Fuzzy logic process with the Compressor recycle system is represented in Fig. 5.

The Mamdani Fuzzy interface system (FIS) is used to create the FLC. The two fuzzy variables are used in the FLC: one is PID output $\left(\mathrm{O}_{\text {pid }}\right)$, and another is Change in PID output $\left(\mathrm{CO}_{\text {pid }}\right)=\mathrm{O}_{\text {pid }}(\mathrm{i})-\mathrm{O}_{\text {pid }}(\mathrm{i}-1)$. Where $\mathrm{i}$ value is set to 1 to 3 .
The two Fuzzy inputs $\left(\mathrm{O}_{\text {pid }}\right.$ and $\left.\mathrm{CO}_{\text {pid }}\right)$ and FLC output (Oflc) are normalized to the display range [0 to 0.6$]$ using fuzzy set membership functions for the given universe of discourse. The Fuzzy Input and output use the triangular-shaped membership functions. The Three fuzzy variables are used as Opid and COpid, which includes Negative Big (NB), Zero (ZO), and Positive Big (PB). Human knowledge is used to create the decision logic for fuzzy rule sets. The Fuzzy rule set for Compressor recycle system is tabulated in Table I. The fuzzy variables like $\mathrm{NB}$ are in the range of [-0.3 to 0.3$], \mathrm{ZO}$ is in the field of [0 to 0.6], and PB is in the range of [0.3 to 0.6]. The nine- rules are used in the fuzzy inputs $\left(\mathrm{O}_{\text {pid }}\right.$ and $\left.\mathrm{CO}_{\text {pid }}\right)$ with three fuzzy variables. The rule set converts these two fuzzy inputs into single FLC output using the centroid technique in the de-fuzzification process.

\section{B. Neuro-Fuzzy Controller (NFC)}

The FLC is extended with NFC using an Artificial Neurofuzzy interface system (ANFIS). The NFC automatically realizes the FIS using Neural networks (NNs). The NFC provides a conversion mechanism (Crisp to fuzzy and viceversa) efficiently. The NFC uses the NN to optimize the FIS using the ANFIS system. The operation of the NFC is represented in Fig. 6. The NN has a learning skill set and is capable of training and testing the FIS in NFC. The NFC uses two inputs like PID controller output $\left(\mathrm{O}_{\text {pid }}\right)$ and change in PID controller output $\left(\mathrm{CO}_{\text {pid }}\right)$ for training. Once the training data is loaded in NFC, generate the FIS using grid partition using the Gaussian membership function. The seven MF's are considered with constant output in NFC.

Next, Train the FIS using the hybrid optimization method. The error tolerance is set to 3 epochs to complete the ANFIS training. Lastly, test the training data to analyze the NFC output.

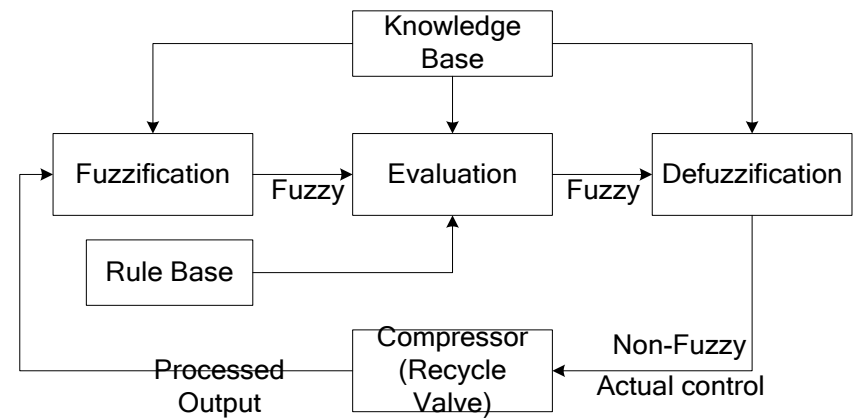

Fig. 5. Fuzzy Logic Process with Compressor Recycle System.

TABLE I. THE FUZZY RULE SET FOR COMPRESSOR RECYCLE SYSTEM

\begin{tabular}{|c|c|c|c|}
\hline E/CE & NB & ZO & PB \\
\hline NB & NB & NB & ZO \\
\hline ZO & NB & ZO & PB \\
\hline PB & ZO & PB & PB \\
\hline
\end{tabular}

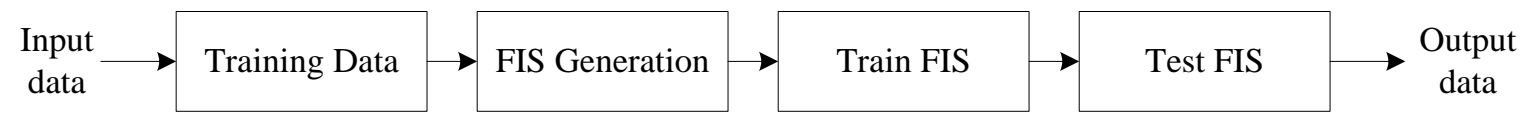

Fig. 6. Operation of NFC. 


\section{Neutral Network Predictive Controller (NNPC)}

The NNPC mainly contains the NN model, Optimization unit, and Compressor recycle system. The NNPC predicts the future performance of the Compressor recycle system using the NN model. The NNPC Based Compressor recycle system for the anti-surge controlling mechanism is represented in Fig. 7. The NNPC is used to calculate the NN model input (m) and further optimize the Compressor recycle system's performance over a defined time horizon.

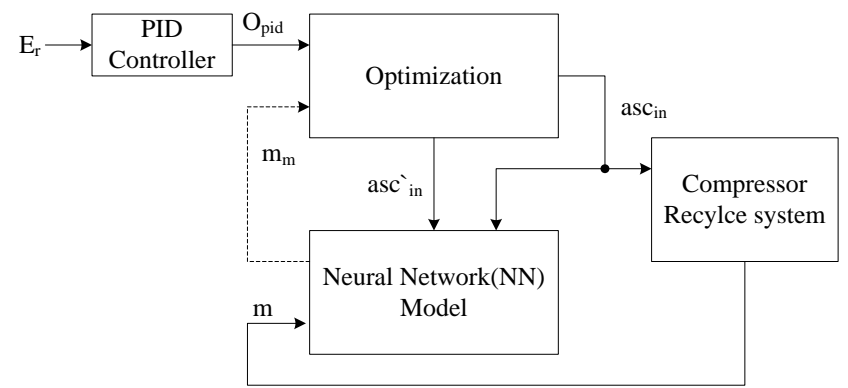

Fig. 7. NNPC based Compressor Recycle System for Anti-surge Control.

The NNPC first identifies the Compressor recycle system (plant) and then uses this model to predict the future performance using a controlling mechanism. In the first stage, the NN model is trained by a predictive control mechanism to analyze the dynamics of the Compressor recycle system. The NN training signal is generated by calculating the predictive error between the NN mode output and Compressor recycle system output. The NN model [26] uses the previous inputs (Optimization) and the previous compressor system's output to analyze the Compressor recycle system's output values. In the second stage, the optimization algorithm [27-28] is used for precision and to calculate the control signal. This control signal minimizes the below Equation's (18) performance over a defined time horizon.

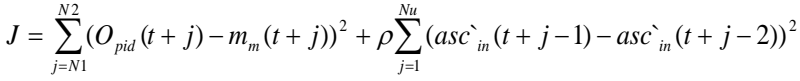

Where $a s c^{\prime}{ }_{i n}$ is a temporary control signal, $\mathrm{O}_{\text {pid }}$ is PID controller's output acts as a desired response, $\mathrm{m}_{\mathrm{m}}$ is the response of NN model, $\rho$ is used to calculate the performance index's sum with squares of control increments. $\mathrm{N}_{1}, \mathrm{~N}_{2}$, and $\mathrm{N}_{\mathrm{u}}$ values define cost and control horizon.

The $\mathrm{J}$ value is minimized using the optimization unit by calculating the $a s c^{\prime}{ }_{i n}$ and inputting the ascin to the Compressor recycle system. The Recycle valve output is represented after the anti-surge controlling mechanism using NNPC controller is described in Equation (19) as follows:

$m_{r}=O_{p i d} \cdot a s c_{i n} \cdot \sqrt{P_{2}-P_{1}}$

The NNPC uses four input layers, five hidden layers, and 1output layer for training the data. The 100 training samples with a sample interval of one second are selected for training the data in the NN training tool. The NNPC obtains the six iterations (epoch) with 8.44e-6 performance and six validation checks while training the data.

\section{RESUltS AND DisCUSSION}

The Compressor recycle system with an anti-surge control mechanism using a PID controller with different controllers is presented in this section. The Simulation results of NNPC based compressor recycle system are introduced and compared with other controllers like PID controller, FLC, and NFC to realize the performance metrics. The NNPC based Compressor recycle system is simulated with the following assumption. The mass flow $(\mathrm{m})$ and compressor speed $(\omega)$ is set to zero initially; two volumes of Pressure $\left(\mathrm{V}_{1}\right.$ and $\left.\mathrm{V}_{2}\right)$ are defined as ambient. The higher mass flow will be obtained by opening the recycle valve. When more mass flow occurs, the operating point (OP) positions are shifted to the right position in $\psi \mathrm{c}$. The simulation results of the Compressor recycle system are represented in Fig. 8. The compressor output mass flow (m), throttle valve output $\left(\mathrm{m}_{\mathrm{t}}\right)$, recycles valve output $\left(\mathrm{m}_{\mathrm{r}}\right)$, and feed flow valve output $\left(\mathrm{m}_{\mathrm{f}}\right)$ is represented on the left-hand side. The torque drive's speed $(\omega)$, suction pressure $\left(\mathrm{P}_{1}\right)$, and Plenum pressure $\left(\mathrm{P}_{2}\right)$ are presented on the right side of Fig. 8.
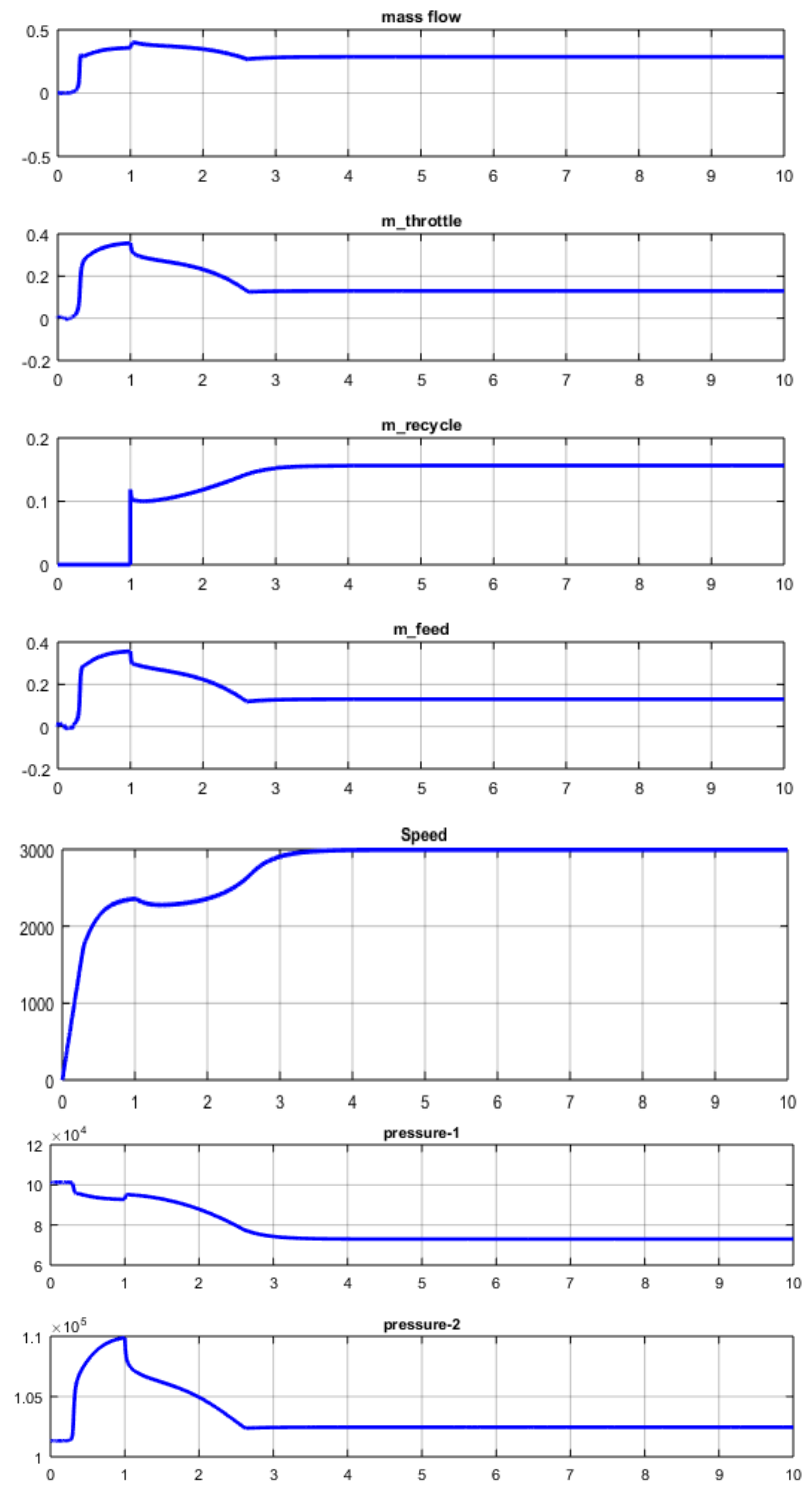

Fig. 8. Simulation Results of Compressor Recycle System. 
The mass flow is set to zero initially and varied based on speed, Throttle, and feed flow response. The throttle output (mt) is changed based on plenum pressure $\left(\mathrm{P}_{2}\right)$ and ambient condition $\left(\mathrm{P}_{01 a}\right)$. The recycle output is not activated until the controller responds and varies based on NNPC controlling mechanism. The feed flow $\left(\mathrm{m}_{\mathrm{f}}\right)$ is zero initially and increases to some extent and gradually decreases, which indicates the system enters into surge line (SL). When the feed flow $\left(m_{f}\right)$ output is steady, the OP's are shifted from SL to SCL. The torque drive provides $3000 \mathrm{rad} / \mathrm{s}$ - speed output $(\omega)$ after the controller response. The two pressures $\left(\mathrm{P}_{1}\right.$ and $\left.\mathrm{P}_{2}\right)$ outputs are decreased eventually and reach steady-state after NNPC response.

The operating point (OP) position and SL and SCL using different controllers in the Compressor recycle system is represented in Fig. 9. The current OP position is identified using other controllers (PID, FLC, NFC, and NNPC) and
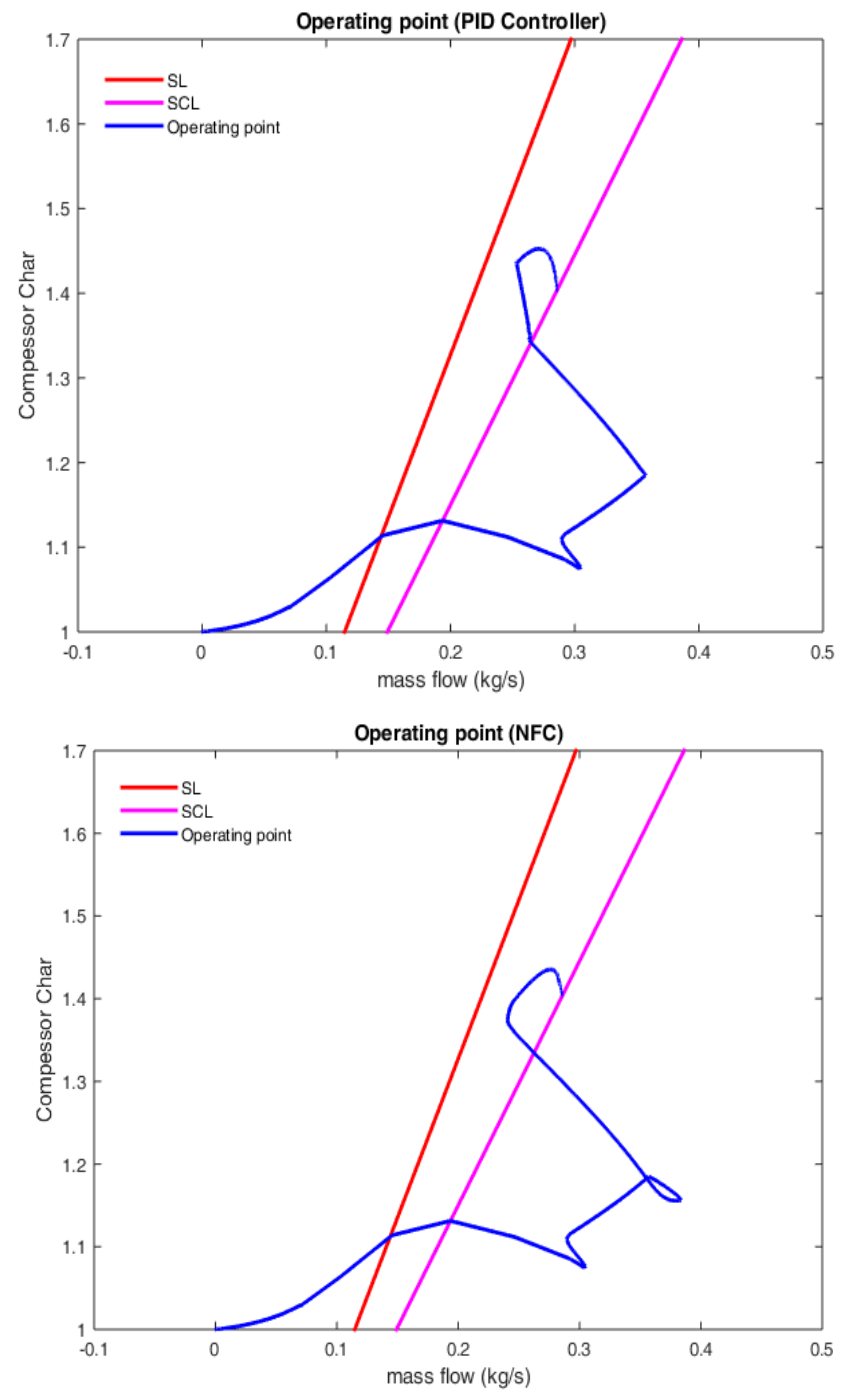

compared with SL and SCL. The SL and SCL are defined initially and perform the simulation of the Compressor recycle system using different controllers to obtain the OP's position.

The SL and SCL are linear and defined with $i$ and $j$ values using Equation (15). The OP is in the left position during the initialization of the Compressor recycle system. Later shifted to the right next to the SCL and moving around it. The OP is very near to SL and turns back to SCL using PID Controller, whereas, in FLC and NFC, The OP is flat and back to SCL. In contrast, The NNPC provides a better OP position, and OP is located next to the SCL. So, the NNPC provides a better OP position than PID, FLC, and NFC in Compressor recycle system.

The different controller's output response for Compressor recycle system is represented in Fig. 10. The output response of all the controllers is initially zero and later varied based on the controlling mechanism in Compressor recycle system.
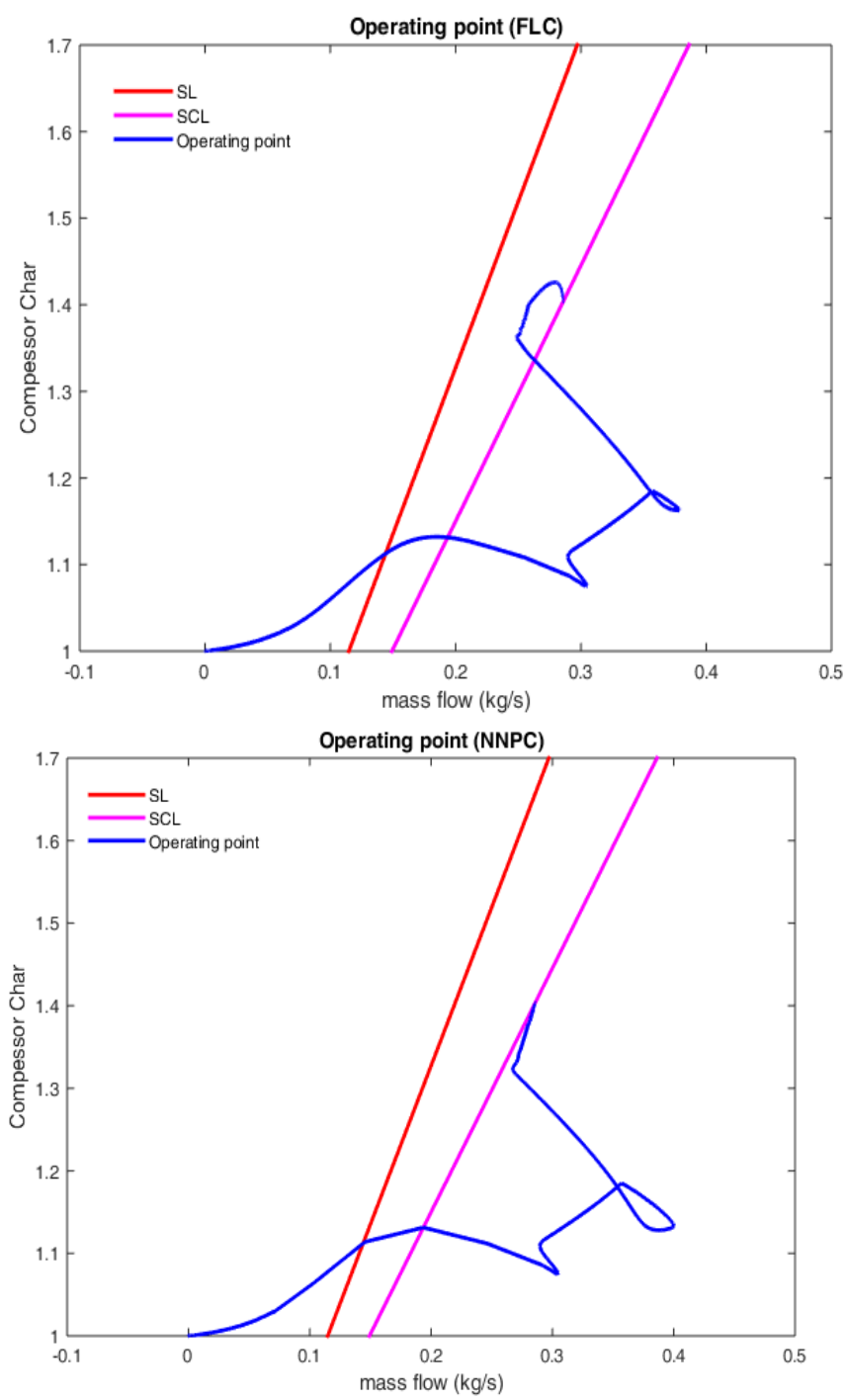

Fig. 9. Different Controller's Operating Points Position in Compressor Recycle System. 


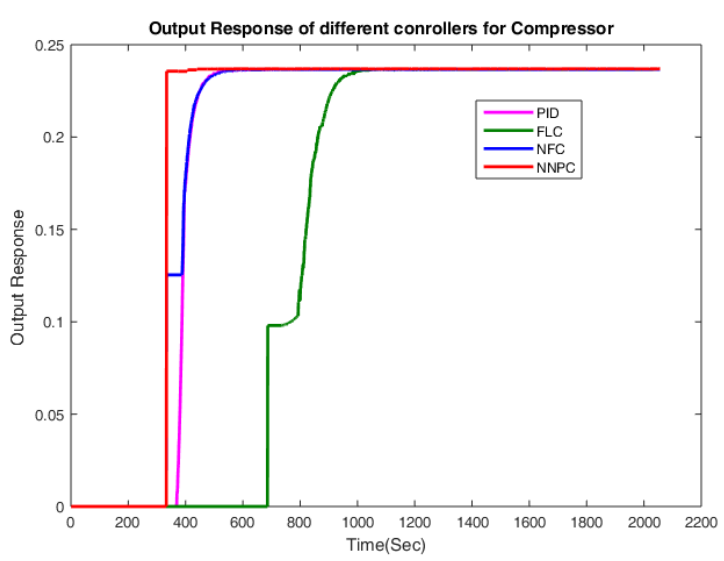

Fig. 10. Dynamic Response of different Controllers for Compressor Recycle System.

The FLC takes more time to provide the output response, whereas NNPC takes less time to provide output response than PID and NFC. The dynamic output response values of different controllers concerning time for the Compressor recycle system are represented in Fig. 11. The output response of the PID controller takes a rise time of $0.976 \mathrm{sec}$, settled at $4.27 \mathrm{sec}$ with an overshoot (\%) of 0.263 sec. Similarly, the FLC controller takes a rise time of $1.905 \mathrm{sec}$, settled at $3.87 \mathrm{sec}$ with an overshoot (\%) time of 0.077 sec. The NFC takes 0.1112 for rising time, settled at $0.236 \mathrm{sec}$ with overshoot (\%) time of zero. In contrast, the output response of the NNPC takes a rise time of $0.013 \mathrm{sec}$, settled at $0.2369 \mathrm{sec}$ with an overshoot (\%) of zero. Overall, the NNPC provides a better dynamic output response with less rise time, settled early, and zero overshoot time than PID, FLC, and NFC.

The error analysis of different controllers for the Compressor recycle system is represented in Fig. 12. The error analysis includes Integral Square Error (ISE), Integral Absolute Error (IAE), and integral time absolute error (ITAE) parameters. The error response of the NNPC based Compressor recycle system improves $14.49 \%$ in ISE, $51.9 \%$ in IAE, and $95.6 \%$ in ITAE than the PID-based Compressor recycle system. Similarly, the $4.83 \%$ in ISE, $34.8 \%$ in IAE, and 91.04 $\%$ in ITAE are improved compared to FLC. Compared to NFC, the $11.86 \%$ in ISE, $34.92 \%$ in IAE and $95.53 \%$ in ITAE are improved in NNPC based compressor recycle system.

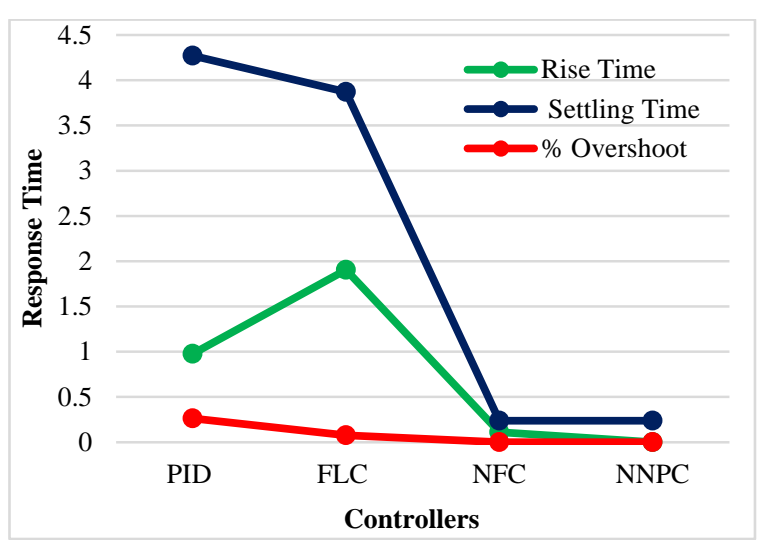

Fig. 11. Output Response Values of different Controllers for Compressor Recycle System.

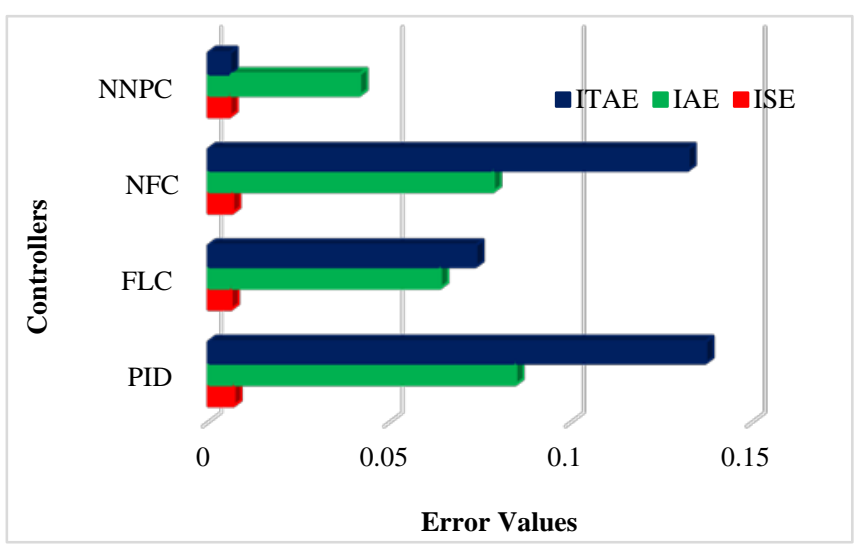

Fig. 12. Error Calculation of different Controllers for Compressors Recycle System.

\section{CONCLUSION AND FUTURE WORK}

In this manuscript, an efficient NNPC based anti-surge control mechanism is modeled for variable-speed centrifugal Compressor recycle system. The NNPC provides higher mass flow in the recycle valve to the compressor system and avoids the surge with better stabilization. The Compressor is working at a more increased mass flow with low Pressure to avoid the surge. The simulation results of NNPC based Compressor recycle systems like mass flow, speed, and Pressure waveforms are discussed. The operating point's position with the Surge line and surge control line for different controllers is represented. The NNPC based mechanism provides dynamic response than PID, FLC, and NFC controller-based mechanisms. The dynamic responses (Rise time, settling time, and \% overshoot time) of the NNPC based Compressor recycle system reduces the ISE of $14.49 \%, 4.83 \%$, and $11.86 \%$ in PID, FLC, and NFC based Compressor recycle system. Similarly, NNPC also reduces the IAE and ITAE values than PID, FLC, and NFC-based controller mechanisms. In future, incorporate the proposed work with real-time gas plants to realize the performance metrics. Also extend the recycle system with new approach to analyze the stability of the compressor system.

\section{REFERENCES}

[1] Gravdahl, Jan Tommy, and Olav Egeland. Compressor surge and rotating stall: Modeling and control. Springer Science \& Business Media, 1999.

[2] Fink, David Alan, Nicholas A. Cumpsty, and Edward M. Greitzer. "Surge dynamics in a free-spool centrifugal compressor system." (1992): 321-332.

[3] Greitzer, Edward M. "Surge and rotating stall in axial flow compressors-Part II: experimental results and comparison with theory." (1976): 199-211.

[4] Gravdahl, Jan Tommy, and Olav Egeland. "A Moore-Greitzer axial compressor model with spool dynamics." In Proceedings of the 36th IEEE Conference on Decision and Control, vol. 5, pp. 4714-4719. IEEE, 1997.

[5] Badmus, O. O., C. N. Nett, and F. J. Schork. "An integrated, full-range surge control/rotating stall avoidance compressor control system." In 1991 American Control Conference, pp. 3173-3180. IEEE, 1991.

[6] Batson, Brett W. "Invariant coordinate systems for compressor control." In Turbo Expo: Power for Land, Sea, and Air, vol. 78729, p. V001T01A070. American Society of Mechanical Engineers, 1996.

[7] Gravdahl, Jan Tommy, Olav Egeland, and Svein Ove Vatland. "Drive torque actuation in active surge control of centrifugal compressors." Automatica 38, no. 11 (2002): 1881-1893. 
[8] Bøhagen, Bjørnar, and Jan Tommy Gravdahl. "Active surge control of compression system using drive torque." Automatica 44, no. 4 (2008): 1135-1140.

[9] Shehata, Raef S., Hussein A. Abdullah, and Fayez FG Areed. "Fuzzy logic surge control in constant speed centrifugal compressors." In 2008 Canadian Conference on Electrical and Computer Engineering, pp. 000653-000658. IEEE, 2008.

[10] Albehigi, Abdulkareem A. Wahab, and Rasha Hyder Hashim. "Experimental and Theoretical Analysis of the Surge in a Centrifugal Compressor." In Modern Methods of Construction Design, pp. 317-328. Springer, Cham, 2014.

[11] Budinis, S., and N. F. Thornhill. "Control of centrifugal compressors via model predictive control for enhanced oil recovery applications." IFACpapers online 48, no. 6 (2015): 9-14.

[12] Budinis, Sara, and Nina F. Thornhill. "Supercritical fluid recycle for surge control of CO2 centrifugal compressors." Computers \& Chemical Engineering 91 (2016): 329-342.

[13] Cortinovis, Andrea, Hans Joachim Ferreau, Daniel Lewandowski, and Mehmet Mercangöz. "Safe and efficient operation of centrifugal compressors using linearized MPC." In 53rd IEEE Conference on Decision and Control, pp. 3982-3987. IEEE, 2014.

[14] Cortinovis, A., H. J. Ferreau, D. Lewandowski, and M. Mercangöz. "Experimental evaluation of MPC-based anti-surge and process control for electric driven centrifugal gas compressors." Journal of process control 34 (2015): 13-25.

[15] Sheng, Hanlin, Wei Huang, Tianhong Zhang, and Xianghua Huang. "Compressor instability active control via a closed-coupled valve and throttle actuators." International Journal of Turbo \& Jet-Engines 32, no. 3 (2015): 257-264.

[16] Gritli, Wafa, Hajer Gharsallaoui, and Mohamed Benrejeb. "PID-type fuzzy scaling factors tuning using genetic algorithm and Simulink design optimization for an electronic throttle valve." In 2016 International Conference on Control, Decision and Information Technologies (CoDIT), pp. 216-221. IEEE, 2016.

[17] Azeem, Mohammad Fazle, and Abdul Kareem. "A fuzzy logic-based super-twisting sliding mode control scheme for electronic throttle control." In 2016 IEEE International Conference on Fuzzy Systems (FUZZ-IEEE), pp. 485-492. IEEE, 2016.

[18] Saeed, Muhammad Ahsan, Nisar Ahmed, Mujahid Hussain, and Adnan Jafar. "A comparative study of controllers for optimal speed control of the hybrid electric vehicle." In 2016 International Conference on Intelligent Systems Engineering (ICISE), pp. 1-4. IEEE, 2016.
[19] Mustafa, Mohamed Iqbal Mohamed, Joseph Xavier Rayappan, and Kanakaraj Jagannathan. "A neuro-fuzzy controller for grid-connected heavy-duty gas turbine power plants." Turkish Journal of Electrical Engineering \& Computer Sciences 25, no. 3 (2017): 2375-2387.

[20] Liying, Zhang, Xu Ting, Li Xuejun, Li Liyan, Guo Pan, and Zhu Yubao. "Study on Nonlinear Compensation Control Method for Electronic Throttle Valve." In 2018 Chinese Automation Congress (CAC), pp. 1085-1089. IEEE, 2018.

[21] Taleb Ziabari, Masoud, Mohammad Reza Jahed-Motlagh, Karim Salahshoor, Amin Ramezani, and Ali Moarefianpur. "Robust adaptive control of surge instability in constant speed centrifugal compressors using tube-MPC." Cogent Engineering 4, no. 1 (2017): 1339335.

[22] Abo-khsheem, K. A. "Active Control of Surge Compressor System." Journal of Electrical \& Electronic Systems 7, no. 3 (2018): 1000267.

[23] Guan, Xudong, Jin Zhou, Chaowu Jin, Yuanping Xu, and Hengbin Cui. "Influence of different operating conditions on centrifugal compressor surge control with active magnetic bearings." Engineering Applications of Computational Fluid Mechanics 13, no. 1 (2019): 824-832.

[24] ARIBI, Yacine, Razika Zammoum Boushaki, and Hocine Loubar. "Active Surge Control of the Recycle Compression System By Hybrid Adaptive Controller." In 2019 4th International Conference on Power Electronics and their Applications (ICPEA), pp. 1-9. IEEE, 2019.

[25] Ebrahimi, Seyed Hossain, and Ahmad Afshari. "An Artificial Neural Network Model for Prediction of the Operational Parameters of Centrifugal Compressors: An Alternative Comparison Method for Regression." Journal of Sciences, Islamic Republic of Iran 31, no. 3 (2020): 259-275.

[26] Asgari, Hamid, XiaoQi Chen, Mohammad B. Menhaj, and Raazesh Sainudiin. "Artificial neural network-based system identification for a single-shaft gas turbine." Journal of Engineering for Gas Turbines and Power 135, no. 9 (2013).

[27] Zhong, Lulu, Yang Liu, Jun Zhao, and Wei Wang. "Deep predictive controller designed for centrifugal compressor system anti-surge." In 2020 Chinese Automation Congress (CAC), pp. 6554-6559. IEEE, 2020.

[28] Shestopalov, Mikhail Yu, Ruslan I. Smirnov, and Damir H. Imaev. "Approximation of the Natural Gas Pumping Compressor Characteristics using a Multi-layer Neural Network." In 2021 IEEE Conference of Russian Young Researchers in Electrical and Electronic Engineering (ElConRus), pp. 1088-1091. IEEE, 2021. 